\title{
Chirality Multiplication and Efficient Chirality Transfer in exo- and endo-Radical Cyclization Reactions of 4-(4'-lodobutyl)quinolones
}

\author{
Martina Dressel and Thorsten Bach $^{1}$
}

Lehrstuhl für Organische Chemie I, Technische Universität München, Lichtenbergstr. 4, 85747 Garching, Germany

\section{Supporting Information}

1. Synthesis of Substrate 3a<smiles>Cc1cc(=O)[nH]c2ccccc12</smiles>

1. ${ }^{n} \mathrm{BuLi},-78^{\circ} \mathrm{C} \rightarrow 0{ }^{\circ} \mathrm{C}$

2. 1 OTBDMS

(THF)<smiles>CCCOCCCCc1cc(=O)[nH]c2ccccc12</smiles>

2 a $39 \%$<smiles>CCOC(=O)c1cccc2[nH]c(=O)cc(CCCCO)c12</smiles>

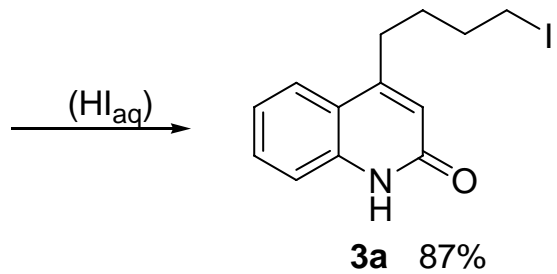

\section{4-[4'-(tert-Butyl-dimethyl-silanyloxy)-butyl]-1H-quinolin-2-one (2a)}

To a suspension of 4-methyl-1H-quinolin-2-one $(2.00 \mathrm{~g}, 12.6 \mathrm{mmol})$ in THF $(60 \mathrm{~mL}),{ }^{n} \mathrm{BuLi}$ (11.5 mL, $27.6 \mathrm{mmol}, 2.40 \mathrm{M}$ in hexane) was added dropwise at $-78^{\circ} \mathrm{C}$ under argon atmosphere. The reaction mixture was allowed to warm to room temperature and stirred for $2 \mathrm{~h}$. The solution was then cooled to $0{ }^{\circ} \mathrm{C}$ and tert-butyl-(3-iodopropoxy)-dimethyl-silane $(5.67 \mathrm{~g}, 18.9 \mathrm{mmol})$ was added. After stirring for $15 \mathrm{~min}$ at room temperature, the reaction mixture was diluted with $20 \mathrm{~mL} 2 \mathrm{~N} \mathrm{HCl}$. Extraction with $\mathrm{CHCl}_{3}(3 \times 20 \mathrm{~mL})$ was followed by washing of the organic extracts with $\mathrm{Na}_{2} \mathrm{CO}_{3}(10 \% \mathrm{w} / \mathrm{w}, 15 \mathrm{~mL})$ and brine $(15 \mathrm{~mL})$, drying with $\mathrm{Na}_{2} \mathrm{SO}_{4}$, and removal of the solvent in vacuo. The crude product was purified by flash chromatography $(6 \times 22 \mathrm{~cm}$, ethyl acetate/pentane $=80 / 20)$ to give a white solid $(1.61 \mathrm{~g}, 39 \%)$.

$\mathrm{C}_{19} \mathrm{H}_{29} \mathrm{NO}_{2} \mathrm{Si}$ $331.52 \mathrm{~g} \mathrm{~mol}^{-1}$<smiles>CCCCOCCCCc1cc(=O)[nH]c2ccccc12</smiles>

1 thorsten.bach@ch.tum.de 
$\boldsymbol{R}_{f}: 0.50$ (ethyl acetate)

mp.: $128^{\circ} \mathrm{C}$

IR (KBr): $\widetilde{v}=3434 \mathrm{~cm}^{-1}$ (br), 2951 (m), $2856(\mathrm{~m}), 1665$ (vs), 1437 (m), $1254(\mathrm{w}), 1103(\mathrm{~m})$.

${ }^{1}$ H-NMR: $\left(360 \mathrm{MHz}\right.$, DMSO-d $\left.{ }^{6}\right): \delta=11.59(\mathrm{~s}, 1 \mathrm{H}), 7.74\left(\mathrm{~d},{ }^{3} \mathrm{~J}=8.1 \mathrm{~Hz}, 1 \mathrm{H}\right), 7.48$ (virt. t, $\left.{ }^{3} J \cong 8.2 \mathrm{~Hz}, 1 \mathrm{H}\right), 7.30\left(\mathrm{~d},{ }^{3} J=8.2 \mathrm{~Hz}, 1 \mathrm{H}\right), 7.17$ (virt. $\left.\mathrm{t},{ }^{3} J \cong 8.0 \mathrm{~Hz}, 1 \mathrm{H}\right), 6.34(\mathrm{~s}, 1 \mathrm{H}), 3.63(\mathrm{t}$, $\left.{ }^{3} J=6.0 \mathrm{~Hz}, 2 \mathrm{H}\right), 2.81\left(\mathrm{t},{ }^{3} J=7.7 \mathrm{~Hz}, 2 \mathrm{H}\right), 1.71-1.63(\mathrm{~m}, 2 \mathrm{H}), 1.60-1.53(\mathrm{~m}, 2 \mathrm{H}), 0.84(\mathrm{~s}, 9 \mathrm{H})$, $0.01(\mathrm{~s}, 6 \mathrm{H})$.

${ }^{13}$ C-NMR: (90.6 MHz, DMSO-d ${ }^{6}$ ): $\delta=161.5$ (s), 151.5 (s), 138.9 (s), 130.0 (d), 124.3 (d), 121.4 (d), $119.8(\mathrm{~d}), 118.6(\mathrm{~s}), 115.6(\mathrm{~d}), 62.0(\mathrm{t}), 31.7(\mathrm{t}), 30.8(\mathrm{t}), 25.7(\mathrm{q}), 24.8(\mathrm{t}), 17.8(\mathrm{~s}),-5.4(\mathrm{q})$.

HRMS: $\quad \mathrm{C}_{19} \mathrm{H}_{29} \mathrm{NO}_{2} \mathrm{Si}\left(\mathrm{M}^{+}{ }^{t} \mathrm{Bu}\right)$

calcd.: $\quad 274.12634$

found: 274.12624

\section{4-(4'-Hydroxybutyl)-1H-quinolin-2-one}

To a solution of $2 \mathbf{a}(1.40 \mathrm{~g}, 4.22 \mathrm{mmol})$ in ethyl acetate $(25 \mathrm{~mL})$ was added TBAF $(8.45 \mathrm{~mL}$, $8.45 \mathrm{mmol}, 1.00 \mathrm{M}$ in THF) at room temperature. After stirring for $14 \mathrm{~h}$, silica gel was added, the solvent removed in vacuo and the crude product purified by flash chromatography $(4 \times 11 \mathrm{~cm}$, ethyl acetate/acetone $=60 / 40)$. The product was obtained as a white solid $(832 \mathrm{mg}, 91 \%)$.

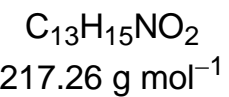<smiles>O=c1cc(CCCCO)c2ccccc2[nH]1</smiles>

$\boldsymbol{R}_{\boldsymbol{f}}: 0.19$ (ethyl acetate/methanol $\left.=95 / 5\right)$

mp.: $142^{\circ} \mathrm{C}$

IR (KBr): $\widetilde{v}=3400 \mathrm{~cm}^{-1}$ (s), 3284 (br), 2939 (m), 2884 (m), 1662 (vs), 1429 (m), 1057 (m), 857 (m), $764(\mathrm{~s})$.

${ }^{1}$ H-NMR: $\left(360 \mathrm{MHz}\right.$, DMSO-d $\left.{ }^{6}\right): \delta=11.57(\mathrm{~s}, 1 \mathrm{H}), 7.75\left(\mathrm{~d},{ }^{3} \mathrm{~J}=8.1 \mathrm{~Hz}, 1 \mathrm{H}\right), 7.48$ (virt. t, $\left.{ }^{3} J \cong 8.3 \mathrm{~Hz}, 1 \mathrm{H}\right), 7.30\left(\mathrm{~d},{ }^{3} J=8.2 \mathrm{~Hz}, 1 \mathrm{H}\right), 7.18$ (virt. $\left.\mathrm{t},{ }^{3} J \cong 8.2 \mathrm{~Hz}, 1 \mathrm{H}\right), 6.35(\mathrm{~s}, 1 \mathrm{H}), 4.40(\mathrm{t}$, $\left.{ }^{3} J=5.2 \mathrm{~Hz}, 1 \mathrm{H}\right), 3.47-3.42(\mathrm{~m}, 2 \mathrm{H}), 2.80\left(\mathrm{t},{ }^{3} \mathrm{~J}=7.7 \mathrm{~Hz}, 2 \mathrm{H}\right), 1.70-1.61(\mathrm{~m}, 2 \mathrm{H}), 1.56-1.48(\mathrm{~m}$, $2 \mathrm{H})$.

${ }^{13}$ C-NMR: (90.6 MHz, DMSO-d ${ }^{6}: \delta=161.6$ (s), 151.7 (s), 139.0 (s), 130.1 (d), 124.4 (d), 121.6 $(\mathrm{d}), 119.9(\mathrm{~d}), 118.7(\mathrm{~s}), 115.6(\mathrm{~d}), 60.3(\mathrm{t}), 32.1(\mathrm{t}), 31.0(\mathrm{t}), 25.2(\mathrm{t})$. 
HRMS: $\quad \mathrm{C}_{13} \mathrm{H}_{15} \mathrm{NO}_{2}\left(\mathrm{M}^{+}\right)$

calcd.: $\quad 217.11028$

found: 217.11018

\section{4-(4'-Iodobutyl)-1H-quinolin-2-one (3a)}

4-(4'-Hydroxybutyl)-1H-quinolin-2-one (600 mg, $2.76 \mathrm{mmol}$ ) was suspended in $15 \mathrm{~mL}$ aqueous hydrogen iodide $(67 \% \mathrm{w} / \mathrm{w})$ and refluxed for $3 \mathrm{~h}$. The crude product precipitated from the reaction mixture when cooled down to room temperature. After filtration, the crude product was recrystallized from ethanol. Compound 3a was obtained as yellow crystals (788 mg, 87\%).

$$
\begin{gathered}
\mathrm{C}_{13} \mathrm{H}_{14} \mathrm{INO}_{2} \\
327.16 \mathrm{~g} \mathrm{~mol}^{-1}
\end{gathered}
$$<smiles>O=c1cc(CCCCI)c2ccccc2[nH]1</smiles>

$\boldsymbol{R}_{f}: 0.23$ (ethyl acetate)

mp.: $192{ }^{\circ} \mathrm{C}$

IR (KBr): $\widetilde{v}=3424 \mathrm{~cm}^{-1}$ (br), 2969 (s), 1684 (vs), 1245 (s), 1163 (s), 1032 (w), 756 (w).

${ }^{1}$ H-NMR: $\left(360 \mathrm{MHz}\right.$, DMSO-d $\left.{ }^{6}\right): \delta=11.61(\mathrm{~s}, 1 \mathrm{H}), 7.76\left(\mathrm{~d},{ }^{3} \mathrm{~J}=8.0 \mathrm{~Hz}, 1 \mathrm{H}\right), 7.48$ (virt. t, $\left.{ }^{3} J \cong 8.3 \mathrm{~Hz}, 1 \mathrm{H}\right), 7.31\left(\mathrm{~d},{ }^{3} J=8.2 \mathrm{~Hz}, 1 \mathrm{H}\right), 7.18$ (virt. $\left.\mathrm{t},{ }^{3} J \cong 8.2 \mathrm{~Hz}, 1 \mathrm{H}\right), 6.36(\mathrm{~s}, 1 \mathrm{H}), 3.33(\mathrm{t}$, $\left.{ }^{3} J=6.9 \mathrm{~Hz}, 2 \mathrm{H}\right), 2.82\left(\mathrm{t},{ }^{3} \mathrm{~J}=7.6 \mathrm{~Hz}, 2 \mathrm{H}\right), 1.90-1.83(\mathrm{~m}, 2 \mathrm{H}), 1.74-1.65(\mathrm{~m}, 2 \mathrm{H})$.

${ }^{13}$ C-NMR: $\quad\left(90.6 \mathrm{MHz}, \mathrm{DMSO}-\mathrm{d}^{6}\right): \delta=161.6$ (s), 151.2 (s), 138.9 (s), 130.1 (d), 124.4 (d), $121.6(\mathrm{~d}), 120.0(\mathrm{~d}), 118.6(\mathrm{~s}), 115.6(\mathrm{~d}), 32.6(\mathrm{t}), 30.0(\mathrm{t}), 29.4(\mathrm{t}), 8.4(\mathrm{t})$.

HRMS: $\quad \mathrm{C}_{13} \mathrm{H}_{14} \mathrm{INO}_{2}\left(\mathrm{M}^{+}\right) \quad$ calcd.: 327.01202

found: 327.01133 
2. Synthesis of Substrate $\mathbf{3 b}$<smiles>Cc1cc(=O)[nH]c2ccccc12</smiles>

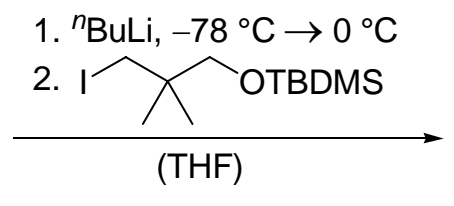<smiles>CCOCC(C)(C)CCc1cc(=O)[nH]c2ccccc12</smiles><smiles>CC(C)(CO)CCc1cc(=O)[nH]c2ccccc12</smiles>

1. $\mathrm{MsCl}, \mathrm{NEt}_{3}, 0^{\circ} \mathrm{C}$ 2. Nal, reflux (DMF)<smiles>CC(C)(CI)CCc1cc(=O)[nH]c2ccccc12</smiles>

\section{3-(tert-Butyl-dimethyl-silanyloxy)-2,2-dimethylpropan-1-ol}

To a solution of 3-(tert-butyl-dimethyl-silanyloxy)-2,2-dimethylpropan-1-ol (4.00 g, $16.2 \mathrm{mmol}$ ) in $100 \mathrm{~mL}$ THF, were added imidazole $(1.65 \mathrm{~g}, 24.3 \mathrm{mmol})$ and triphenylphosphine $(6.37 \mathrm{~g}$, $24.3 \mathrm{mmol})$ under argon. After stirring for $10 \mathrm{~min}$, iodine $(4.52 \mathrm{~g}, 17.8 \mathrm{mmol})$ was added to the reaction mixture and stirring was continued for $14 \mathrm{~h}$ at room temperature. Silica gel was added and the crude product was purified by flash chromatography $(9 \times 15 \mathrm{~cm}$, pentane/diethylether $=$ $90 / 10)$. The product was obtained as a colourless liquid (3.23 g, 61\%).

$$
\begin{gathered}
\mathrm{C}_{11} \mathrm{H}_{25} \mathrm{IOSi} \\
328.31 \mathrm{~g} \mathrm{~mol}^{-1}
\end{gathered}
$$<smiles>CC(C)(CI)CO[SnH3]</smiles>

$\boldsymbol{R}_{\boldsymbol{f}}: 0.86($ pentane/diethylether $=90 / 10)$

IR (film): $\widetilde{v}=2955$ (s), 1471 (s), 1258 (s), 1104 (vs), 837 (s), 776 (m).

${ }^{1}$ H-NMR: $\left(360 \mathrm{MHz}, \mathrm{CDCl}_{3}\right): \delta=3.35(\mathrm{~s}, 2 \mathrm{H}), 3.22(\mathrm{~s}, 2 \mathrm{H}), 1.00(\mathrm{~s}, 6 \mathrm{H}), 0.90(\mathrm{~s}, 9 \mathrm{H}), 0.06$ (s, $6 \mathrm{H})$.

${ }^{13}$ C-NMR: (90.6 MHz, $\mathrm{CDCl}_{3}$ ): $\delta=69.9$ (t), 35.8 (s), 25.9 (q), 23.9 (q), 20.9 (t), 18.2 (s), -5.5 (q).

HRMS: $\quad \mathrm{C}_{11} \mathrm{H}_{25} \mathrm{IOSi}\left(\mathrm{M}^{+}{ }^{t} \mathrm{Bu}\right)$

calcd.: 271.00153

found: 271.00121 


\section{4-[4'-(tert-Butyl-dimethyl-silanyloxy)-3',3'-dimethylbutyl]-1H-quinolin-2-one (2b)}

Compound 2b was prepared in analogy to the synthesis of 2a: 4-methyl-1H-quinolin-2-one $(1.11 \mathrm{~g}, 6.94 \mathrm{mmol})$ was suspended in THF $(30 \mathrm{~mL})$ under argon. After addition of ${ }^{n} \mathrm{BuLi}$ (5.91 mL, $\quad 13.9 \mathrm{mmol}, \quad 2.35 \mathrm{M}$ in hexane) and 3-(tert-butyl-dimethyl-silanyloxy)-2,2dimethylpropan-1-ol $(2.85 \mathrm{~g}, 8.68 \mathrm{mmol})$, the crude product was purified by flash chromatography $(6 \times 12 \mathrm{~cm}$, ethyl acetate $/$ pentane $=80 / 20)$ to afford a white solid $(2.59 \mathrm{mmol}$, $37 \%)$.

$$
\begin{gathered}
\mathrm{C}_{21} \mathrm{H}_{33} \mathrm{NO}_{2} \mathrm{Si} \\
359.58 \mathrm{~g} \mathrm{~mol}^{-1}
\end{gathered}
$$<smiles>CCCOCC(C)(C)CCc1cc(=O)[nH]c2ccccc12</smiles>

$\boldsymbol{R}_{\boldsymbol{f}}: 0.56$ (ethyl acetate)

mp.: $134-136^{\circ} \mathrm{C}$

IR (KBr): $\widetilde{v}=3100 \mathrm{~cm}^{-1}$ (s, br), 2955 (m), 2855 (m), 1652 (vs), 1433 (m), 1247 (w), 1092 (s), $848(\mathrm{~s}), 759(\mathrm{~m})$.

${ }^{1}$ H-NMR: (360 MHz, DMSO-d $\left.{ }^{6}\right): \delta=11.59(\mathrm{~s}, 1 \mathrm{H}), 7.72\left(\mathrm{~d},{ }^{3} \mathrm{~J}=7.9 \mathrm{~Hz}, 1 \mathrm{H}\right), 7.48$ (virt. t, $\left.{ }^{3} J \cong 7.3 \mathrm{~Hz}, 1 \mathrm{H}\right), 7.31\left(\mathrm{~d},{ }^{3} J=7.9 \mathrm{~Hz}, 1 \mathrm{H}\right), 7.18$ (virt. $\left.\mathrm{t},{ }^{3} J \cong 7.3 \mathrm{~Hz}, 1 \mathrm{H}\right), 6.33(\mathrm{~s}, 1 \mathrm{H}), 3.37$ (s, 2H), 2.76-2.71 (m, 2H), 1.55-1.50 (m, 2H) $0.93(\mathrm{~s}, 6 \mathrm{H}), 0.89(\mathrm{~s}, 9 \mathrm{H}), 0.05(\mathrm{~s}, 6 \mathrm{H})$.

${ }^{13}$ C-NMR: (90.6 MHz, DMSO-d $\left.{ }^{6}\right): \delta=161.5$ (s), 152.5 (s), 138.9 (s), 130.0 (d), 124.1 (d), 121.4 (d), $119.7(\mathrm{~d}), 118.6(\mathrm{~s}), 115.6(\mathrm{~d}), 70.2(\mathrm{t}), 38.0(\mathrm{t}), 35.1(\mathrm{t}), 30.3(\mathrm{t}), 26.4(\mathrm{~s}), 25.7(\mathrm{q}), 23.8(\mathrm{q})$, $17.8(\mathrm{~s}),-5.6(\mathrm{q})$.

HRMS: $\quad \mathrm{C}_{21} \mathrm{H}_{33} \mathrm{NO}_{2} \mathrm{Si}\left(\mathrm{M}^{+}{ }^{t} \mathrm{Bu}\right) \quad$ calcd.: 302.15762

found: 302.15764

\section{4-(4'-Hydroxy-3',3'-dimethylbutyl)-1H-quinolin-2-one}

To a stirred solution of compound $\mathbf{2 b}(800 \mathrm{mg}, 2.22 \mathrm{mmol})$ in $15 \mathrm{~mL}$ of ethyl acetate was added TBAF (4.44 mL, $4.44 \mathrm{mmol}, 1.00 \mathrm{M}$ in THF). The reaction mixture was stirred for $14 \mathrm{~h}$ and after complete conversion, silica gel was added and the solvent removed in vacuo. The crude product was purified by flash chromatography $(3 \times 10 \mathrm{~cm}$, ethyl acetate). The product was obtained as a white solid (535 mg, 98\%).

$$
\begin{gathered}
\mathrm{C}_{15} \mathrm{H}_{19} \mathrm{NO}_{2} \\
245.32 \mathrm{~g} \mathrm{~mol}^{-1}
\end{gathered}
$$<smiles>CC(C)(CO)CCc1cc(=O)[nH]c2ccccc12</smiles> 
$\boldsymbol{R}_{f}: 0.31$ (ethyl acetate)

mp.: $221^{\circ} \mathrm{C}$

IR (KBr): $\widetilde{v}=3342 \mathrm{~cm}^{-1}$ (s, br), 2954 (m), 2865 (m), 1651 (vs), 1431 (m), 1247 (w), 1069 (s), $869(\mathrm{~m}), 750(\mathrm{~s})$.

${ }^{1}$ H-NMR: $\left(360 \mathrm{MHz}\right.$, DMSO-d $\left.{ }^{6}\right): \delta=11.56(\mathrm{~s}, 1 \mathrm{H}), 7.75\left(\mathrm{~d},{ }^{3} \mathrm{~J}=8.0 \mathrm{~Hz}, 1 \mathrm{H}\right), 7.46$ (virt. t, $\left.{ }^{3} J \cong 7.1 \mathrm{~Hz}, 1 \mathrm{H}\right), 7.31\left(\mathrm{~d},{ }^{3} J=8.2 \mathrm{~Hz}, 1 \mathrm{H}\right), 7.21$ (virt. t, $\left.{ }^{3} J \cong 8.1 \mathrm{~Hz}, 1 \mathrm{H}\right), 6.36(\mathrm{~s}, 1 \mathrm{H}), 3.21(\mathrm{~d}$, $\left.{ }^{2} J=5.3 \mathrm{~Hz}, 2 \mathrm{H}\right), 2.77-2.72(\mathrm{~m}, 2 \mathrm{H}), 1.63-1.53(\mathrm{~m}, 2 \mathrm{H}), 0.91(\mathrm{~s}, 6 \mathrm{H})$.

${ }^{13}$ C-NMR: (90.6 MHz, DMSO-d ${ }^{6}$ ): $\delta=161.6$ (s), 152.7 (s), 138.9 (s), 130.0 (d), 124.2 (d), 121.5 $(\mathrm{d}), 119.7(\mathrm{~d}), 118.6(\mathrm{~s}), 115.6(\mathrm{~d}), 69.3(\mathrm{t}), 35.0(\mathrm{t}), 30.4(\mathrm{t}), 26.4(\mathrm{~s}), 23.9(\mathrm{q})$.

HRMS: $\quad \mathrm{C}_{15} \mathrm{H}_{19} \mathrm{NO}_{2}\left(\mathrm{M}^{+}\right) \quad$ calcd.: 245.14159

found: 245.14139

\section{4-(4'-Iodo-3',3'-dimethylbutyl)-1H-quinolin-2-one}

A solution of 4-(4-hydroxy-3',3'-dimethylbutyl)- $1 H$-quinolin-2-one (500 $\mathrm{mg}, 2.04 \mathrm{mmol}$ ) in $20 \mathrm{~mL}$ dimethylformamide was cooled to $0{ }^{\circ} \mathrm{C}$ under argon. After adding triethylamine $(423 \mu \mathrm{L}$, $309 \mathrm{mg}, 3.06 \mathrm{mmol})$, methanesulfonyl chloride $(178 \mu \mathrm{L}, 256 \mathrm{mg}, 2.24 \mathrm{mmol})$ was added dropwise and the reaction mixture was stirred for $3 \mathrm{~h}$ at $0{ }^{\circ} \mathrm{C}$. Water $(10 \mathrm{~mL})$ and ethyl acetate $(20 \mathrm{~mL})$ were added and the layers were separated. The aqueous phase was extracted with ethyl acetate $(3 \times 15 \mathrm{~mL})$ and the combined organic extracts were washed with brine $(10 \mathrm{~mL})$, dried with $\mathrm{Na}_{2} \mathrm{SO}_{4}$ and the solvent evaporated in vacuo. The crude product was dissolved again in $30 \mathrm{~mL}$ dimethylformamide and sodium iodide $(927 \mathrm{mg}, 6.20 \mathrm{mmol})$ was added. The reaction mixture was refluxed for $14 \mathrm{~h}$ in the dark and after cooling to room temperature, water $(50 \mathrm{~mL})$ and ethyl acetate $(50 \mathrm{~mL})$ were added. The aqueous phase was extracted with ethyl acetate $(3 \times 20 \mathrm{~mL})$ and the combined organic extracts were washed with brine $(20 \mathrm{~mL})$ and dried with $\mathrm{Na}_{2} \mathrm{SO}_{4}$. The solvent was removed in vacuo and the crude product purified by flash chromatography $(4 \times 10 \mathrm{~cm}$, ethyl acetate/pentane $=80 / 20)$. The pure product was obtained as a pale yellow solid (319 $\mathrm{mg}, 58 \%)$.<smiles>CC(C)(CI)CCc1cc(=O)[nH]c2ccccc12</smiles>

$\boldsymbol{R}_{\boldsymbol{f}}: 0.45$ (ethyl acetate)

mp.: $146^{\circ} \mathrm{C}$

IR (KBr): $\widetilde{v}=3351 \mathrm{~cm}^{-1}$ (s, br), 2961 (s), 2853 (s), 1654 (vs), 1555 (m), 1436 (m), 755 (m). 
${ }^{1}$ H-NMR: $\left(360 \mathrm{MHz}\right.$, DMSO-d $\left.{ }^{6}\right): \delta=11.60(\mathrm{~s}, 1 \mathrm{H}), 7.78\left(\mathrm{~d},{ }^{3} J=8.0 \mathrm{~Hz}, 1 \mathrm{H}\right), 7.47$ (virt. t, ${ }^{3} J \cong$ $8.3 \mathrm{~Hz}, 1 \mathrm{H}), 7.32\left(\mathrm{~d},{ }^{3} \mathrm{~J}=7.4 \mathrm{~Hz}, 1 \mathrm{H}\right), 7.20$ (virt. t, $\left.{ }^{3} \mathrm{~J} \cong 7.0 \mathrm{~Hz}, 1 \mathrm{H}\right), 6.37$ (s, 1H), $3.41(\mathrm{~s}, 2 \mathrm{H})$, 2.77-2.72 (m, 2H), 1.64-1.59 (m, 2H), $1.11(\mathrm{~s}, 6 \mathrm{H})$.

${ }^{13}$ C-NMR: (90.6 MHz, DMSO-d ${ }^{6}$ ): $\delta=161.5$ (s), 151.8 (s), 138.9 (s), 130.1 (d), 124.2 (d), 121.5 (d), $119.7(d), 118.5(\mathrm{~s}), 115.6(\mathrm{~d}), 40.0(\mathrm{~s}), 33.4(\mathrm{t}), 26.4(\mathrm{t}), 26.1(\mathrm{q}), 24.9(\mathrm{t})$.

HRMS: $\quad \mathrm{C}_{15} \mathrm{H}_{18} \mathrm{INO}\left(\mathrm{M}^{+}\right) \quad$ calcd.: 355.04331

found: $\quad 355.04325$

\section{Radical Cyclization Reactions}<smiles>O=c1cc(CCCCI)c2ccccc2[nH]1</smiles><smiles>[13CH3][13CH3]</smiles><smiles>O=C1Nc2ccccc2[C@H]2CCCC[C@H]12</smiles>

trans-4<smiles>O=C1Nc2ccccc2[C@H]2CCCC[C@H]12</smiles>

cis-4

\section{6a,7,8,9,10,10a-Hexahydrophenantridin-6(5H)-one (4)}

To a solution of iodide $3 \mathbf{a}(50.0 \mathrm{mg}, 0.15 \mathrm{mmol})$ and chiral complexing agent $1(0.25-$ 2.50 equivalents) in $20 \mathrm{~mL}$ of the given solvent, $\mathrm{Bu}_{3} \mathrm{SnH}(79.4 \mu \mathrm{L}, 87.3 \mathrm{mg}, 0.30 \mathrm{mmol})$ and $\mathrm{BEt}_{3}\left(75 \mu \mathrm{L}, 0.075 \mathrm{mmol}, 1.00 \mathrm{M}\right.$ in hexane) were added at room temperature or at $0{ }^{\circ} \mathrm{C}$. Additional $\mathrm{BEt}_{3}(75 \mu \mathrm{L}, 0.075 \mathrm{mmol}, 1.00 \mathrm{M}$ in hexane) was added after $24 \mathrm{~h}$. After complete conversion ( $2 \mathrm{~d}$ ), silica gel was added and the solvent removed in vacuo. The crude product was purified by flash chromatography $(2 \times 8 \mathrm{~cm}$, diethylether/pentane $=70 / 30)$. Products 4 were obtained as a mixture of diastereoisomers (see table 1). The diastereoisomers trans- $\mathbf{4}$ and cis-4 were separated by reverse-phase HPLC (YMC ODS-A, $250 \times 20 \mathrm{~mm} \mathrm{i.d}$, gradient $=30 / 70 \rightarrow 60 / 40$ acetonitrile/water over $30 \mathrm{~min}$ ). The enantiomeric excess was determined by chiral GC (2,3-di-O-methyl-6-O-TBDMS- $\beta$-cyclodextrin-column).

$$
\mathrm{C}_{13} \mathrm{H}_{15} \mathrm{NO}
$$
$201.26 \mathrm{~g} \mathrm{~mol}^{-1}$<smiles>O=C1Nc2ccccc2[C@H]2CCCC[C@@H]12</smiles>

trans-diastereoisomer:

$\boldsymbol{R}_{\boldsymbol{f}}: 0.42$ (diethylether/pentane $\left.=70 / 30\right)$

${ }^{1}$ H-NMR: $\left(360 \mathrm{MHz}, \mathrm{CDCl}_{3}\right): \delta=7.74(\mathrm{~s}, 1 \mathrm{H}), 7.25-7.16(\mathrm{~m}, 2 \mathrm{H}), 7.07$ (virt. t, ${ }^{3} \mathrm{~J} \cong 7.6 \mathrm{~Hz}$, $1 \mathrm{H}), 6.76\left(\mathrm{~d},{ }^{3} \mathrm{~J}=7.8 \mathrm{~Hz}, 1 \mathrm{H}\right), 2.682 .60(\mathrm{~m}, 1 \mathrm{H}), 2.54-2.50(\mathrm{~m}, 1 \mathrm{H}), 2.47-2.39(\mathrm{~m}, 1 \mathrm{H}), 2.13-$ $2.05(\mathrm{~m}, 1 \mathrm{H}), 1.98-1.94(\mathrm{~m}, 2 \mathrm{H}), 1.44-1.26(\mathrm{~m}, 4 \mathrm{H})$. 
${ }^{13}$ C-NMR: (90.6 MHz, DMSO-d ${ }^{6}$ ): $\delta=173.1$ (s), 136.6 (s), 128.4 (s), 127.4 (d), 124.4 (d), 123.0 $(\mathrm{d}), 114.9(\mathrm{~d}), 43.3(\mathrm{~d}), 37.9(\mathrm{~d}), 28.8(\mathrm{t}), 26.2(\mathrm{t}), 25.2(\mathrm{t}), 25.2(\mathrm{t})$.

(6aR,10aR)-enantiomer (trans-4): $\mathrm{GC}\left(160{ }^{\circ} \mathrm{C} \rightarrow 200{ }^{\circ} \mathrm{C}\right.$ at $\left.0.5^{\circ} \mathrm{C} / \mathrm{min}\right): t_{\mathrm{R}}=43.13 \mathrm{~min}$. (6aS,10aS)-enantiomer (trans-ent-4): $\mathrm{GC}\left(160{ }^{\circ} \mathrm{C} \rightarrow 200^{\circ} \mathrm{C}\right.$ at $\left.0.5^{\circ} \mathrm{C} / \mathrm{min}\right): t_{\mathrm{R}}=43.66 \mathrm{~min}$.

The spectroscopic data were in accordance with reported data. ${ }^{[2,3,4]}$

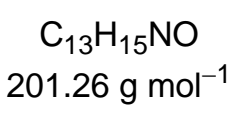<smiles>O=C1Nc2ccccc2[C@H]2CCCC[C@H]12</smiles>

cis-diastereoisomer:

$\boldsymbol{R}_{\boldsymbol{f}}: 0.42$ (diethylether/pentane $\left.=70 / 30\right)$

${ }^{1}$ H-NMR: $\left(360 \mathrm{MHz}, \mathrm{CDCl}_{3}\right): \delta=8.00(\mathrm{~s}, 1 \mathrm{H}), 7.17-7.13(\mathrm{~m}, 2 \mathrm{H}), 6.99$ (virt. t, ${ }^{3} \mathrm{~J} \cong 7.7 \mathrm{~Hz}$, $1 \mathrm{H}), 6.73\left(\mathrm{~d},{ }^{3} \mathrm{~J}=7.7 \mathrm{~Hz}, 1 \mathrm{H}\right), 2.94$ (virt. dt, ${ }^{3} \mathrm{~J}=10.4 \mathrm{~Hz},{ }^{3} \mathrm{~J} \cong 4.8 \mathrm{~Hz}, 1 \mathrm{H}$ ), 2.80 (virt. q, $\left.{ }^{3} J \cong{ }^{3} J=4.8 \mathrm{~Hz}, 1 \mathrm{H}\right), 1.74-1.46(\mathrm{~m}, 8 \mathrm{H})$.

${ }^{13}$ C-NMR: (90.6 MHz, $\mathrm{CDCl}_{3}$ ): $\delta=172.6$ (s), 136.3 (s), 127.4 (s), 127.3 (d), 123.3 (d), 123.3 (d), $115.1(\mathrm{~d}), 40.5(\mathrm{~d}), 39.3(\mathrm{~d}), 29.7(\mathrm{t}), 25.1(\mathrm{t}), 24.2(\mathrm{t}), 22.9(\mathrm{t})$.

(6aS,10aR)-enantiomer (cis-4): $\mathrm{GC}\left(160^{\circ} \mathrm{C} \rightarrow 200^{\circ} \mathrm{C}\right.$ at $\left.0.5^{\circ} \mathrm{C} / \mathrm{min}\right): t_{\mathrm{R}}=36.66 \mathrm{~min}$. (6aR,10aS)-enantiomer (ent-cis-4): $\mathrm{GC}\left(160{ }^{\circ} \mathrm{C} \rightarrow 200^{\circ} \mathrm{C}\right.$ at $\left.0.5^{\circ} \mathrm{C} / \mathrm{min}\right): t_{\mathrm{R}}=35.97 \mathrm{~min}$.

The spectroscopic data were in accordance with reported data. ${ }^{[2,3,4]}$<smiles>CC(C)(CI)CCc1cc(=O)[nH]c2ccccc12</smiles>

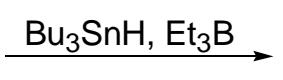<smiles>CC1(C)CCC2(CC(=O)Nc3ccccc32)C1</smiles>

\section{Spiro[3,4-dihydro-1H-quinoline-2-one-4,1',3',3'-dimethyl-cyclopentane] (5)}

To a solution of iodide $3 \mathbf{b}(30.0 \mathrm{mg}, 0.084 \mathrm{mmol})$ and chirale complexing agent $\mathbf{1}(0.25-$ 2.50 equivalents) in $5.50 \mathrm{~mL}$ of the given solvent, $\mathrm{Bu}_{3} \mathrm{SnH}(44.5 \mu \mathrm{L}, 48.9 \mathrm{mg}, 0.17 \mathrm{mmol})$ and

Ninomiya, I.; Yamauchi, S.; Kiguschi, T.; Shinobara, A.; Naito, T., J. Chem. Soc. Perkin. Trans. 1 1974, 17 1747-1751.

Masamune, T.; Takasugi, M.; Suginome, H.; Yokohama, M. J. Org. Chem. 1964, 29, 681-685.

Bach, T.; Grosch, B.; Strassner, T.; Herdtweck, E. J. Org. Chem. 2003, 68, 1107-1116. 
$\mathrm{BEt}_{3}\left(42 \mu \mathrm{L}, 0.042 \mathrm{mmol}, 1.00 \mathrm{M}\right.$ in hexane) were added at room temperature or at $0{ }^{\circ} \mathrm{C}$. Additional $\mathrm{BEt}_{3}(42 \mu \mathrm{L}, 0.042 \mathrm{mmol}, 1.00 \mathrm{M}$ in hexane) was added after $24 \mathrm{~h}$. After complete conversion ( $2 \mathrm{~d}$ ), silica gel was added and the solvent removed in vacuo. The crude product was purified by flash chromatography $(2 \times 6 \mathrm{~cm}$, diethylether/pentane $=50 / 50)$. The cyclization product was obtained as a white solid $(12.4 \mathrm{mg}, 64 \%)$. The enantiomeric excess was determined by chiral GC (2,3-di-O-methyl-6-O-TBDMS- $\beta$-cyclodextrin-column).

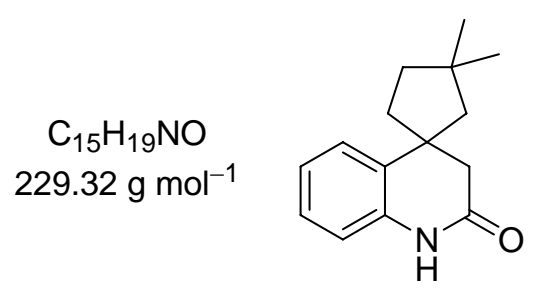

$\boldsymbol{R}_{\boldsymbol{f}}: 0.41$ (diethylether/pentane $\left.=70 / 30\right)$

mp.: $198^{\circ} \mathrm{C}$

IR (KBr): $\widetilde{v}=3351 \mathrm{~cm}^{-1}$ (s, br), 2961 (s), 2853 (s), 1654 (vs), 1555 (m), 1436 (m), 755 (m).

$[\alpha]_{\mathrm{D}}^{20}=+12.5(c=0.43$ in $\mathrm{MeOH})[90 \% e e]$

${ }^{1}$ H-NMR: $\left(360 \mathrm{MHz}\right.$, DMSO-d $\left.\mathrm{d}^{6}\right): \delta=10.05(\mathrm{~s}, 1 \mathrm{H}), 7.21\left(\mathrm{~d},{ }^{3} \mathrm{~J}=7.6 \mathrm{~Hz}, 1 \mathrm{H}\right), 7.15$ (virt. t, $\left.{ }^{3} J \cong 7.7 \mathrm{~Hz}, 1 \mathrm{H}\right), 6.96$ (virt. t, $\left.{ }^{3} \mathrm{~J} \cong 7.5 \mathrm{~Hz}, 1 \mathrm{H}\right), 6.86\left(\mathrm{~d},{ }^{3} \mathrm{~J}=7.8 \mathrm{~Hz}, 1 \mathrm{H}\right), 1.64-1.55(\mathrm{~m}, 8 \mathrm{H})$, $0.89(\mathrm{~s}, 6 \mathrm{H})$.

${ }^{13}$ C-NMR: (90.6 MHz, DMSO-d ${ }^{6}$ ): $\delta=166.8$ (s), 137.5 (s), 131.4 (s), 127.5 (d), 124.0 (d), 121.8 (d), $114.7(d), 38.6(t), 37.3(\mathrm{~s}), 32.7(\mathrm{t}), 29.9(\mathrm{t}), 28.2(\mathrm{~s}), 27.6(\mathrm{t}), 26.1(\mathrm{q}), 26.1(\mathrm{q})$.

HRMS: $\quad \mathrm{C}_{15} \mathrm{H}_{19} \mathrm{NO}\left(\mathrm{M}^{+}\right) \quad$ calcd.: 229.14666

found: 229.14662

$S$-enantiomer (5): $\mathrm{GC}\left(160{ }^{\circ} \mathrm{C} \rightarrow 200^{\circ} \mathrm{C}\right.$ at $\left.0.5^{\circ} \mathrm{C} / \mathrm{min}\right): t_{\mathrm{R}}=51.78 \mathrm{~min}$.

$R$-enantiomer (ent-5): $\mathrm{GC}\left(160^{\circ} \mathrm{C} \rightarrow 200^{\circ} \mathrm{C}\right.$ at $\left.0.5^{\circ} \mathrm{C} / \mathrm{min}\right): t_{\mathrm{R}}=52.18 \mathrm{~min}$. 


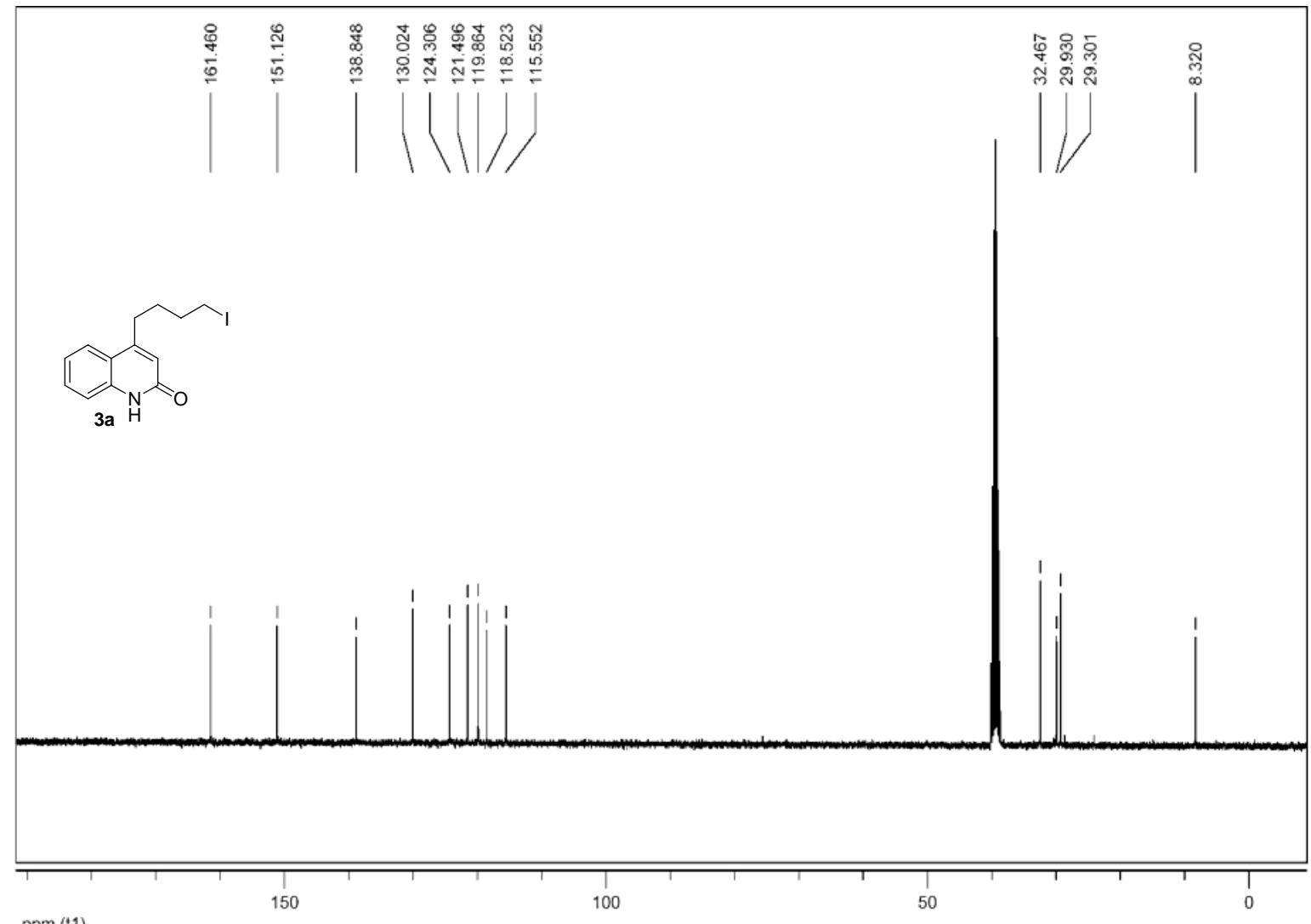

ppm (t1)

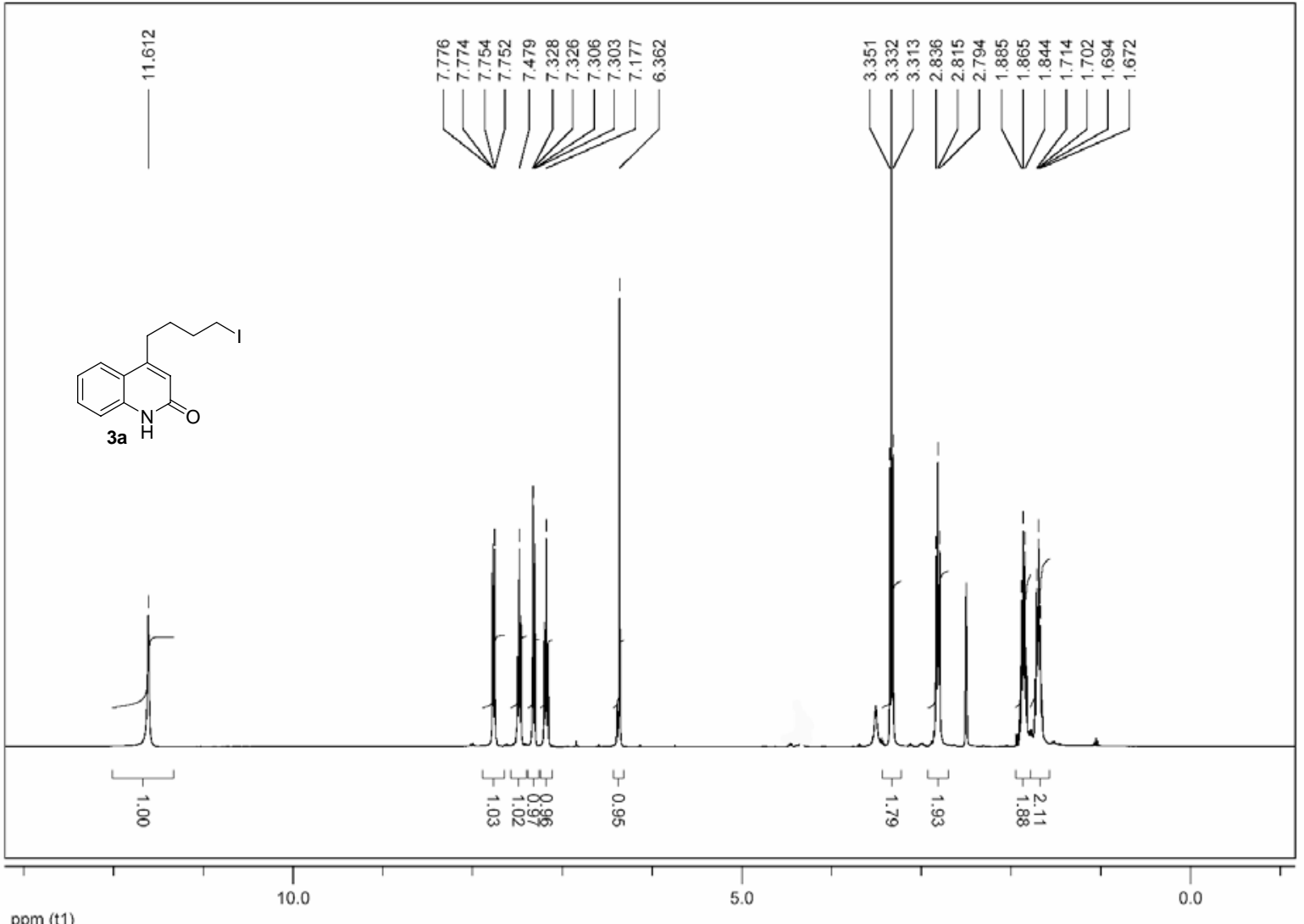



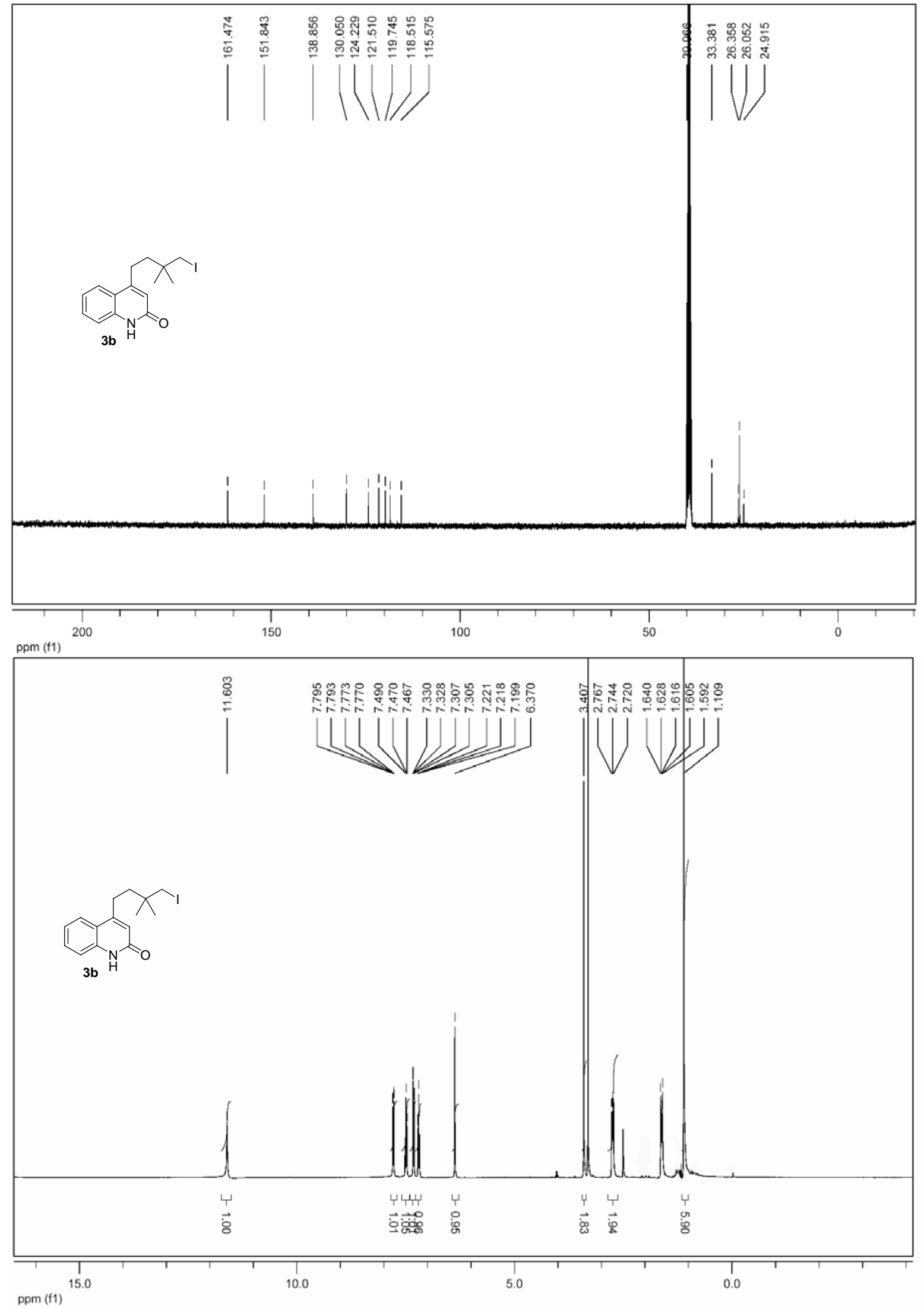

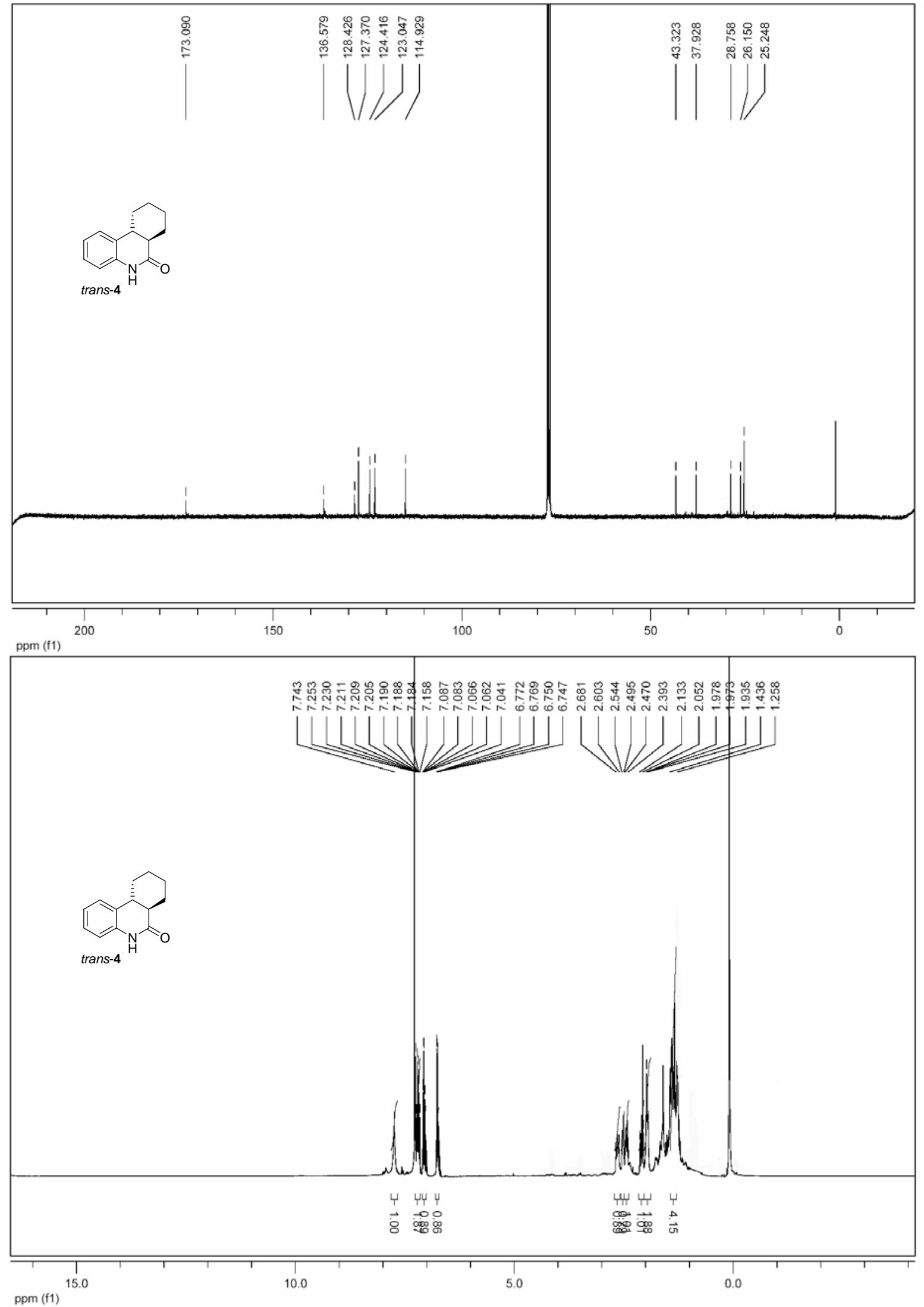


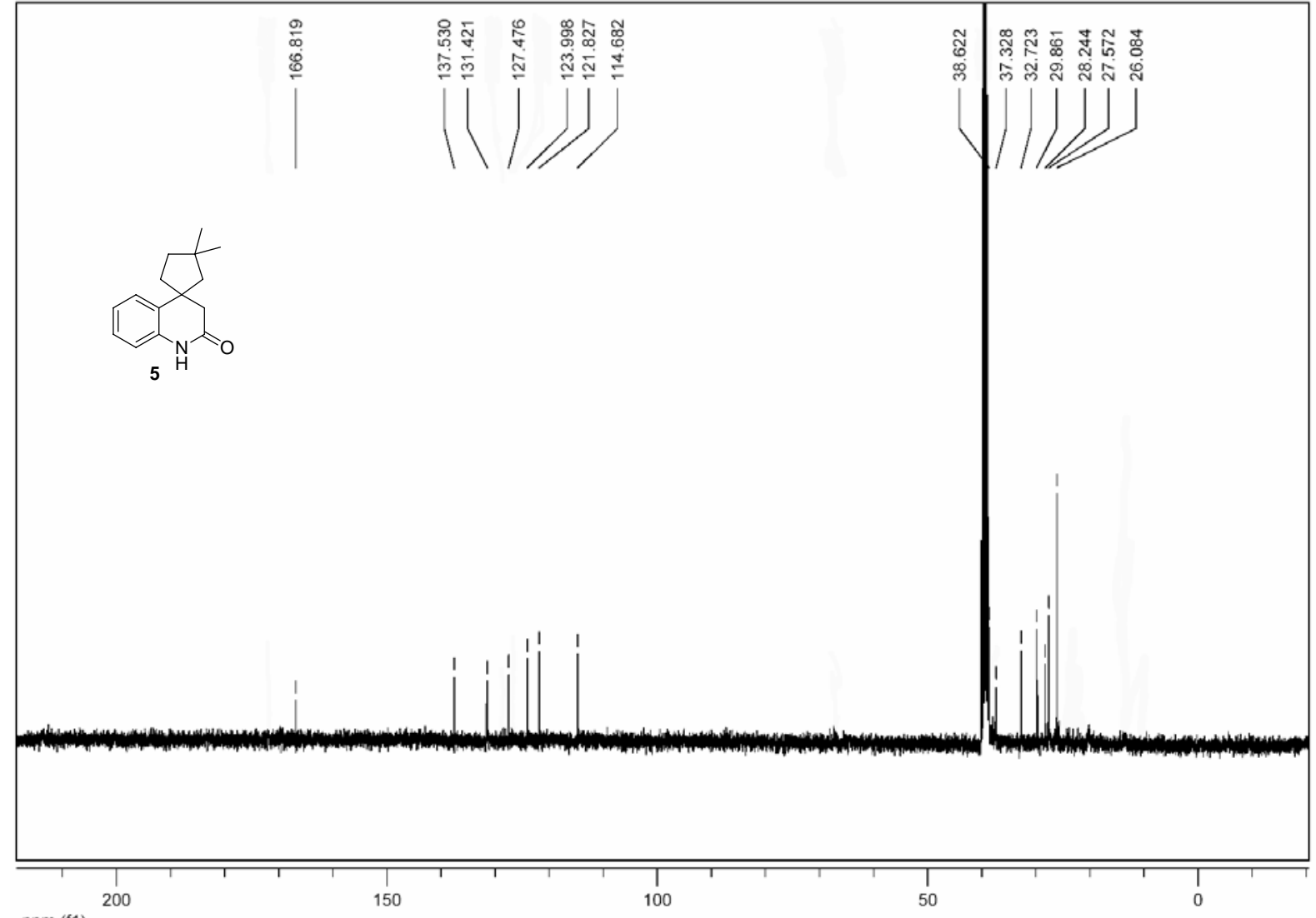
ppm (f1)
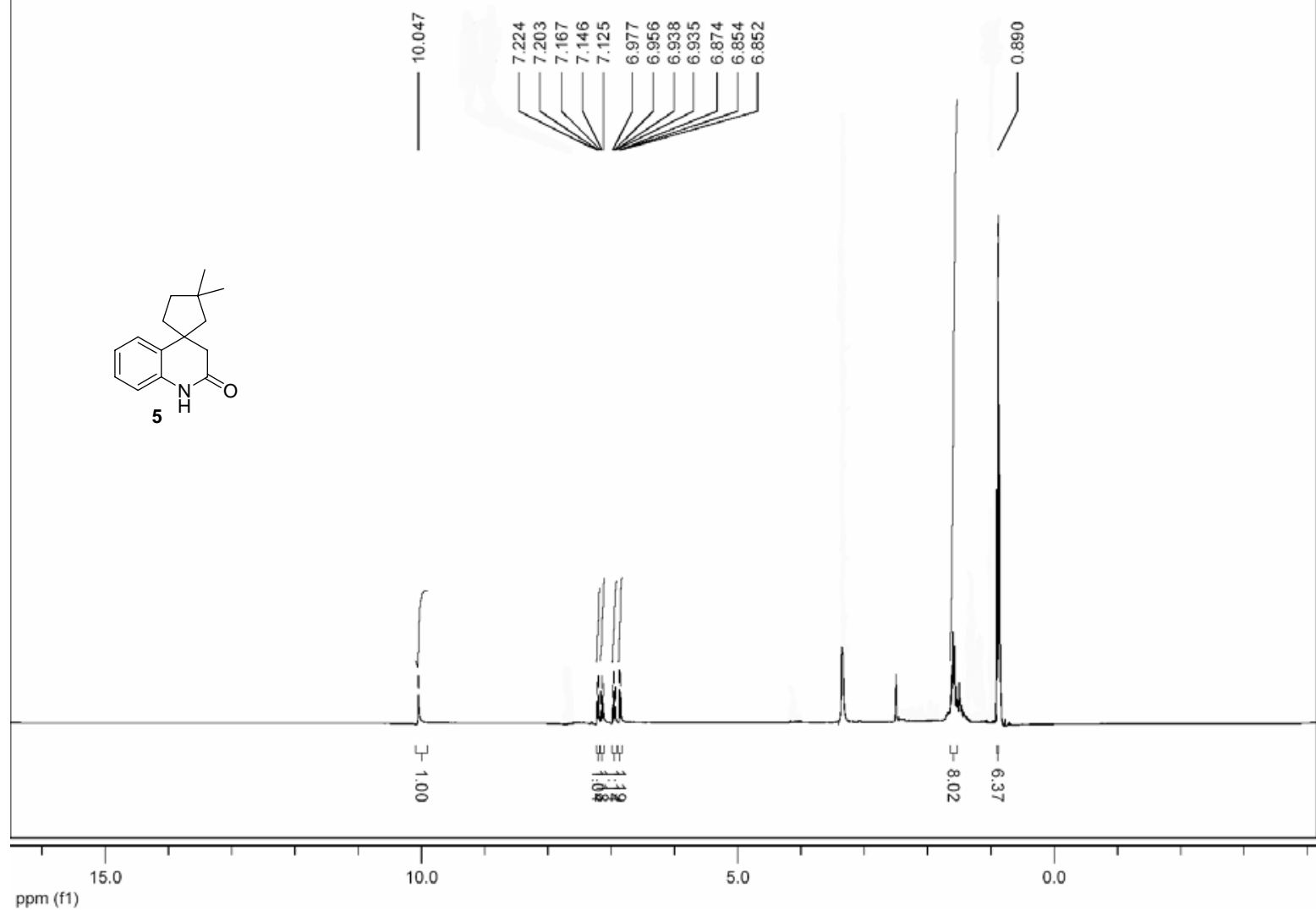